\title{
Modulatory role of vitamin A on the Candida albicans-induced immune response in human monocytes
}

\author{
Tilman E. Klassert • Anja Hanisch • Julia Bräuer • \\ Esther Klaile $\cdot$ Kerstin A. Heyl $\cdot$ Michael M. Mansour • \\ Jenny M. Tam $\cdot$ Jatin M. Vyas $\cdot$ Hortense Slevogt
}

Received: 6 March 2014 / Accepted: 5 August 2014 / Published online: 17 August 2014

(C) The Author(s) 2014. This article is published with open access at Springerlink.com

\begin{abstract}
Beyond its well-documented role in reproduction, embryogenesis and maintenance of body tissues, vitamin A has attracted considerable attention due to its immunomodulatory effects on both the innate and the adaptive immune responses. In infectious diseases, vitamin A has been shown to have a host-protective effect in infections of bacterial, viral or protozoan origin. Nevertheless, its impact in fungal infections remains unknown. Meanwhile, the frequency of invasive mycoses keeps on growing, with Candida albicans being the major opportunistic fungal pathogen and associated with high mortality. In the present work, we explored the impact of all-trans retinoic acid (atRA), the most active metabolite of vitamin A, on the innate immune response against $C$. albicans in human monocytes. Our results show a strong immunomodulatory role for atRA, leading to a significant down-regulation of the fungi-induced expression and secretion of the proinflammatory cytokines TNF $\alpha$, IL6 and IL12. Moreover, atRA significantly suppressed the expression of Dectin-1,
\end{abstract}

Electronic supplementary material The online version of this article (doi:10.1007/s00430-014-0351-4) contains supplementary material, which is available to authorized users.

T. E. Klassert · A. Hanisch · J. Bräuer · E. Klaile · K. A. Heyl ·

H. Slevogt $(\bowtie)$

Septomics Research Center, Jena University Hospital,

Albert-Einstein-Strasse 10, 07745 Jena, Germany

e-mail: hortense.slevogt@med.uni-jena.de

A. Hanisch · E. Klaile

Center for Sepsis Control and Care (CSCC), Jena University

Hospital, Erlanger Allee 101, 07747 Jena, Germany

M. M. Mansour · J. M. Tam · J. M. Vyas

Division of Infectious Diseases, Department of Medicine,

Massachusetts General Hospital, Harvard Medical School,

55 Fruit Street, GRJ-5-504, Boston, MA, USA a major fungal pattern recognition receptor, as well as the Dectin-1-dependent cytokine production. Both RARdependent and RAR-independent mechanisms seem to play a role in the atRA-mediated immunomodulation. Our findings open a new direction to elucidate the role of vitamin A on the immune function during fungal infections.

Keywords Vitamin A - Retinoic acid - Candida albicans . Dectin-1

\section{Introduction}

Vitamin $\mathrm{A}$ is an essential nutrient obtained through the diet either as provitamin-A (carotenoids) or as preformed vitamin A (retinol and retinyl esters) [1, 2]. In its esterified form, it can be stored in the liver, where it is continually hydrolyzed to retinol and deployed into circulation [3, 4]. Once in its target tissues, two dehydrogenases are able to convert the retinol into retinoic acid, the biologically active metabolite of vitamin A [5]. In this form, vitamin A is known to play an essential role in multiple biological processes, including reproduction, embryogenesis, maintenance of body tissues and augmentation of the immune system [6-8]. Over the last decades, an increasing effort has been devoted to better define its involvement in the regulation of the immune response, since vitamin A deficiency (VAD) has been associated with an increased susceptibility to severe infectious diseases [9].

Several in vitro studies have shed light into the role of vitamin A not only as an important factor for normal immune system development, but also as a modulator of both the innate and the adaptive immune responses [4, 10]. Vitamin A has shown to regulate the development of B-lymphocytes and its immunoglobulin production 
[11-13]. In T-cells, retinoic acid is able to attenuate the Th1-associated gene expression and skew the immune response toward a Th2 profile [14]. Retinoic acid is also able to modulate the LPS-induced cytokine and/ or chemokine production in further innate immune cells, including monocytes [15], macrophages and dendritic cells [16]. Moreover, the role of vitamin A as immunomodulator has been reinforced by interventional studies with infants and children with VAD. Here, vitamin A supplementation could reduce the morbidity and/or mortality from measles, malaria and certain forms of diarrhea [17]. These findings are also supported by several in vitro and animal studies, where a host-protective effect of vitamin A could be described for infections involving a bacterial, viral or protozoan origin [18]. Nevertheless, the ability of vitamin A to modulate the immune response against fungal infections is still unknown.

The frequency of invasive mycoses due to opportunistic fungal pathogens has been significantly growing in intensive care units over the last decades [19, 20]. Among invasive fungal infections in humans, Candida albicans remains the most important cause and is associated with high morbidity and mortality [21]. As shown for Candidainduced sepsis in mice models, the fatal host damage results from an exaggerated immune response rather than from the pathogen itself [22]. Therefore, modulation of the immune response might be an interesting strategy to reduce C. albicans-associated immunopathology.

The orchestration of the antifungal response starts with the recognition of the pathogen by immune cells provided with fungal pattern recognition receptors (PRR) [23]. Monocytes express most of the fungal PRRs and have shown to play a particularly important role in the early recognition of the pathogen in invasive candidiasis [23, 24]. Moreover, monocytes are the most effective mononuclear cell-type at killing $C$. albicans, while their cytokine secretion is important for subsequent innate and adaptive immune activation [23, 25]. However, inflammatory monocytes have also been linked to dysregulated immune responses in invasive candidiasis [22]. Thus, modulation of the immune response in monocytes might be of particular importance for the course of infection. Among the PRRs expressed on these innate cells, Dectin-1 has shown to play a prominent role in the immune response against C. albicans, not only mediating phagocytosis but also triggering the oxidative burst and the production of several pro-inflammatory cytokines [26]. Hence, in the present study, we analyzed the immunomodulatory role of vitamin $\mathrm{A}$ on the innate immune response against $C$. albicans with a main focus on the Dectin-1-mediated response. For this purpose, we employed $\beta$-1,3-glucan beads which were designed to serve as "fungal-like particles" eliciting a dominant Dectin-1 response [27, 28].

\section{Materials and methods}

Material

Beta-1,3-glucan beads were prepared as previously described [27]. atRA was purchased from Sigma-Aldrich (Germany) and dissolved in absolute ethanol. The RAR $\alpha$ agonist BMS753, the RAR $\gamma$-agonist BMS961, as well as the RAR $\alpha$ antagonist BMS195614 and the RAR $\gamma$ antagonist MM11253 were purchased from Tocris Bioscience (UK). Monoclonal mouse anti-human Dectin-1 MAB1859 (clone \#259931) antibody was purchased from R\&D Systems (Germany). Mouse $\operatorname{IgG}_{2 B}$ isotype control antibody was purchased from eBioscience (UK). APC-conjugated polyclonal goat anti-mouse antibody and APC-conjugated monoclonal mouse anti-human CD14 antibody were purchased from BD Biosciences (Germany). Polyclonal rabbit anti-Galectin-3 SC-20157 antibody was purchased from Santa Cruz (USA) and polyclonal rabbit anti-Actin (20-33) antibody was purchased from Sigma-Aldrich (Germany). HRP conjugated goat anti-rabbit IgG $(\mathrm{H}+\mathrm{L})$ antibody was purchased from Dianova (Germany).

\section{Candida albicans isolate}

Overnight fungal cultures of the virulent wild-type strain SC5314 [29] were grown in YPD medium, washed three times and resuspended in PBS at a concentration of $10^{8}$ yeasts $/ \mathrm{ml}$. To avoid overbalanced growth of $C$. albicans and monocyte-killing due to hyphae formation, we inactivated the fungal yeasts. UV inactivation of the cells was performed on a UVC-500-Crosslinker (Amersham, UK) using two doses of $100,000 \mu \mathrm{j} / \mathrm{cm}^{2}$ immediately before cell stimulation.

\section{Monocyte isolation}

Human monocytes were isolated from buffy coats kindly provided by Dagmar Barz (Institute of Transfusional Medicine of the Jena University Hospital). Peripheral blood mononuclear cells (PBMCs) were isolated by density gradient centrifugation following manufacturer's instructions. Briefly, blood diluted 1:1 with PBS was layered onto an equal volume of Ficoll-Paque Plus (GE-Healthcare, Germany) and centrifuged in Leukosep Falcon tubes at $800 \times \mathrm{g}$ for $15 \mathrm{~min}$. After centrifugation, the leukocyte band was collected, washed with cold $\mathrm{NaCl} 0.45 \%$ and subjected to erythrocyte lysis using a hypotonic buffer. Cells were then washed twice in cold PBS and counted on a hemocytometer. Cell viability was assessed by trypan blue and propidium iodide/AnnexinV staining. To further isolate the monocytes, we used the monocyte isolation kit II (Miltenyi, UK) which couples negative selection with a cocktail of 
Table 1 Sequence of forward and reverse primers of indicated target genes and the size expected for each PCR product

\begin{tabular}{lllll}
\hline Human gene & Symbol & Forward primer & Reverse primer & Size (bp) \\
\hline Peptidylpropyl isomerase B & $P P I B$ & ATGTAGGCCGGGTGATCTTT & TGAAGTTCTCATCGGGGAAG & 219 \\
Hypoxanthine phosphoribosyltransferase 1 & $H P R T 1$ & GACCAGTCAACAGGGGACAT & AACACTTCGTGGGGTCCTTTTC & 195 \\
Tumor necrosis factor alpha & $T N F \alpha$ & TTCTCCTTCCTGATCGTGGC & ACTCGGGGTTCGAGAAGATG & 150 \\
Interleukin 6 & $I L 6$ & GAGGAGACTTGCCTGGTGAA & TGGGTCAGGGGTGGTTATTG & 186 \\
Interleukin 10 & $I L 10$ & GCTGAGAACCAAGACCCAGA & GCATTCTTCACCTGCTCCAC & 143 \\
Interleukin 12 subunit beta & $I L 12 B$ & ACAACATCTGTTTCAGGGCCA & GGTCCAAGGTCCAGGTGATA & 239 \\
Dectin-1 & $C L E C 7 A$ & ACACTTCGACTCTCAAAGCA & TACAGCAATGAGGCGCCAA & 91 \\
Toll-like receptor 2 & $T L R 2$ & TGCATTCCCAAGACACTGGA & AGGGAGGCATCTGGTAGAGT \\
Galectin-3 & $L G A L S 3$ & CCCATCTTCTGGACAGCCAA & CTTCACCGTGCCCAGAATTG \\
Retinoic acid receptor alpha & $R A R A$ & CCACATGTTCCCCAAGATGC & GCCCTCTGAGTTCTCCAACA & 151 \\
Retinoic acid receptor beta & $R A R B$ & TCGTCTGCCAGGACAAATCA & TTGGCATCGATTCCTGGTGA & 158 \\
Retinoic acid receptor gamma & $R A R G$ & CAAGGTCAGCAAAGCCCATC & ACTTGGTAGCCAGCTCACTG & 137 \\
\hline
\end{tabular}

biotin-conjugated monoclonal antibodies and magnetic cell sorting using the quadro-MACS (Miltenyi, UK). Purity of the obtained monocytes was $>92 \%$ as assessed by CD14labeling and flow cytometric analysis.

\section{Stimulation assays}

After monocyte isolation, cells were resuspended at $4 \times 10^{6}$ cells/ml in RPMI GlutaMax-Medium (Invitrogen, UK) supplemented with $1 \%$ Penicillin/Streptomycin (Invitrogen, UK), plated on 6-well plates (VWR International, Germany) and allowed to equilibrate at $37{ }^{\circ} \mathrm{C}$ for $2 \mathrm{~h}$. Monocytes were then pre-incubated with $1 \mu \mathrm{M}$ of atRA or the specific RAR agonists for $30 \mathrm{~min}$, followed by addition of the previously prepared $C$. albicans yeast at a fungusmonocyte ratio of $1: 1$. This ratio was predetermined in pilot experiments to preserve cell viability while yielding a suitable host gene response. When RAR antagonists were used, these were added 30 min before atRA, at a concentration of $1 \mu \mathrm{M}$. In the stimulation assay using $\beta$-1,3-glucan beads as specific ligands of Dectin-1, a 5:1 ratio was used. The cells were then incubated for 5 or $16 \mathrm{~h}$ at $37{ }^{\circ} \mathrm{C}$ and $5 \%$ $\mathrm{CO}_{2}$. Viability of the monocytes was $>90 \%$, as assessed by trypan blue and propidium iodide-staining. Additionally, AnnexinV staining was used to exclude an increase in apoptotic events. After incubation, the monocytes were harvested for RNA isolation and the culture supernatants were collected and stored at $-80{ }^{\circ} \mathrm{C}$.

\section{RT-PCR and quantitative PCR}

To analyze the gene expression of the target genes, total RNA was isolated from $8 \times 10^{6}$ monocytes using the Qiagen RNeasy mini kit. An additional step was included to remove the residual genomic DNA using DNaseI (Qiagen, Germany). A NanoDrop D-1000 Spectrophotometer
(Thermo-Fisher Scientific, Germany) was then used to assess the amount and quality of the RNA. Complementary DNA (cDNA) was synthesized from 1,5 $\mu \mathrm{g}$ of RNA using the High Capacity cDNA Reverse Transcription Kit (Applied Biosystems, UK) following manufacturer's instructions. For PCR-analysis, specific primers for each target gene were designed using the online primer-BLAST tool of the National Center for Biotechnology Information (NCBI, http://www.ncbi.nlm.nih.gov/tools/primer-blast/). In order to improve the PCR efficiency, possible secondary structures of the amplicons were taken into account by characterizing their nucleotide sequence using the Mfold algorithm [30]. The sequences of all primers used for amplification are listed in Table 1.

PCR of the cDNA was carried out on a S1000TM Thermal Cycler (BioRad, UK) in a $25 \mu$ reaction volume containing $0.2 \mu \mathrm{M}$ primers, $1 \mathrm{U}$ Taq DNA polymerase (5-Prime, UK) and $200 \mu \mathrm{M}$ dNTPs. Thermal conditions included an initial $95{ }^{\circ} \mathrm{C}$ denaturation step for $3 \mathrm{~min}$, and then 35 cycles of $10 \mathrm{~s}$ at $94{ }^{\circ} \mathrm{C}, 30 \mathrm{~s}$ at $60^{\circ} \mathrm{C}$ and $30 \mathrm{~s}$ at $72{ }^{\circ} \mathrm{C}$. The resulting PCR products were separated on an ethidium bromide stained agarose gel and visualized under a UV-transiluminator to confirm the expected amplicon size.

To quantify the relative expression of each gene, a Corbett Rotor-Gene 6000 (Qiagen, Germany) was used as RealTime qPCR apparatus. Each sample was analyzed in duplicate in a total reaction volume of $20 \mu \mathrm{l}$ containing $10 \mu \mathrm{l}$ of $2 \times$ SensiMix SYBR Master Mix (Bioline, UK) and $0.2 \mu \mathrm{M}$ of each primer. All qPCRs were set up using a $C A S-1200$ pipetting robot (Qiagen, Germany). The cycling conditions were $95{ }^{\circ} \mathrm{C}$ for $10 \mathrm{~min}$ followed by 40 cycles of $95{ }^{\circ} \mathrm{C}$ for $15 \mathrm{~s}, 60^{\circ} \mathrm{C}$ for $20 \mathrm{~s}$ and $72{ }^{\circ} \mathrm{C}$ for $20 \mathrm{~s}$. For each experiment, an RT-negative sample was included as control. Specificity of the qPCRs was assessed by melting curve analysis and size verification by electrophoresis. The relative expression of the target genes was analyzed using a modified Pfaffl 
method [31, 32]. To determine significant differences in the mRNA expression between different experimental conditions, the relative quantity (RQ) for each sample was calculated using the formula $1 / \mathrm{E}^{\mathrm{Ct}}$, where $E$ is the efficiency and $\mathrm{Ct}$ the threshold cycle. The RQ was then normalized to the geometric mean of two housekeeping genes: hypoxanthine phosphoribosyltransferase1 (HPRT1) and peptidylpropyl isomerase B (PPIB). The stability of the housekeeping genes was assessed using the BestKeeper algorithm [33]. The normalized RQ (NRQ) values were $\log 2$-transformed for further statistical analysis with GraphPad PRISM v5.0.

\section{Flow cytometry}

To analyze the expression of Dectin-1 on the cell surface, monocytes were washed with PBS containing $10 \%$ FBS and stained with anti-Dectin- 1 antibody $(1 \mu \mathrm{g} / \mathrm{ml}, 30 \mathrm{~min})$ and with APC-conjugated goat anti-mouse $\mathrm{IgG}(2,5 \mu \mathrm{g} / \mathrm{ml}, 30 \mathrm{~min})$. A mouse-IgG $\mathrm{IB}_{2 \mathrm{~B}}$-antibody was used as isotype control. Samples were measured on a FACSAria II apparatus (BD Biosciences; Germany) and data were analyzed using the FLOWJO 7.6.4 software. The resulting mean fluorescence intensities (MFIs) were normalized to those of unstained cells in each case.

\section{Cytokine measurements}

TNF $\alpha$, IL6, IL12 and IL10 secretion by the monocytes was analyzed using commercially available ELISA kits (Human TNF $\alpha$ Elisa Kit, Thermo Scientific; Human IL12 Elisa Kit, Hölzel Diagnostika; Human IL6 Elisa Kit, eBioscience; Human IL10 Elisa Kit, eBioscience) according to the manufacturer's instructions. An Infinite M200 reader (Tecan, UK) was used to measure the optical density (OD), and the concentration of each cytokine was calculated from the respective standard curve by five-parameter logistic analysis using the Magellan v.6 software (Tecan, UK).

\section{Statistical analysis}

Statistical analyses were performed using GraphPad PRISM v5.0 software (San Diego, USA). Two-sided pairwise $t$ test or repeated measures ANOVA with Dunnett's post hoc test were used to determine statistical significance. In all cases, the level of significance was set at $p \leq 0.05$.

\section{Results}

Retinoic acid modulates the cytokine production induced by $C$. albicans and $\beta$-1,3-glucan in human monocytes

We assessed the impact of vitamin $\mathrm{A}$ on the immune response to $C$. albicans by challenging monocytes with
UV-killed yeasts in the absence or presence of $1 \mu \mathrm{M}$ atRA. In a first approach, we analyzed the expression of TNF $\alpha$, IL6, IL12b and IL10 at transcriptional level by Real-Time qPCR. For accurate normalization purposes, we tested the stability of the housekeeping genes among the experimental conditions using the BestKeeper algorithm [33]. Since both PPIB (standard deviation (SD) of the $\mathrm{Ct}=0,48$; coefficient of variance $(\mathrm{CV})$ of the $\mathrm{Ct}=2,60)$ and HPRT1 $(\mathrm{SD}(\mathrm{Ct})=0,49 ; \mathrm{CV}(\mathrm{Ct})=2,22)$ showed a highly stable expression, the geometric mean of both genes was used for further relative expression calculations.

After $5 \mathrm{~h}$ of incubation with $C$. albicans yeasts, we could observe a clear increase in the mRNA expression levels of all four cytokines analyzed (Fig. 1a). However, in the presence of atRA in the cell culture medium, the up-regulation of the pro-inflammatory cytokines was significantly suppressed. As shown in Fig. 1a, the $C$. albicans-mediated expression of $\mathrm{TNF} \alpha$ could be dropped from a 78 -fold expression (in the absence of atRA) to a 21-fold expression when atRA was present. In a similar way, we could observe a significant reduction of 83 and $96 \%$ in the gene expression levels of IL6 and IL12b, respectively (Fig. 1a). This modulation by retinoic acid occurs in a dose-dependent manner, and an inhibitory effect of atRA on all three cytokines could already be observed with concentrations as low as $0,01 \mu \mathrm{M}$ (Suppl. Fig. 1). On the other hand, we could not observe any effect of atRA on the $C$. albicansinduced IL10 expression (Fig. 1a), even if in the absence of fungal challenge, the addition of atRA led to an up-regulation of this anti-inflammatory cytokine (data not shown).

Comparable results were obtained when the monocytes were stimulated with $\beta-1,3$-glucan beads to specifically address the Dectin-1-response (Fig. 1b). As shown in Fig. 1b, the activation of this early fungal-recognition receptor, leading to an up-regulation of all three proinflammatory cytokines, was down-regulated by atRA at transcriptional level. In contrast, the expression of the anti-inflammatory cytokine IL10 was rather potentiated by atRA, although not in a statistically significant manner.

To further confirm our findings at protein level, we analyzed the culture supernatants for the presence of $\mathrm{TNF} \alpha$, IL6, IL12 and IL10 after $16 \mathrm{~h}$ of stimulation with either C. albicans or $\beta$-glucan beads. As shown in Fig. 2, proinflammatory cytokine secretion upon fungal stimulation was severely affected by atRA. Co-stimulation with atRA decreased the amount of secreted TNF $\alpha$, IL6 and IL12 in a significant manner, whereas no effect could be observed on the IL10 release. (Fig. 2a). Similar results were observed when the monocytes were stimulated with the $\beta$-glucan beads. In this case, atRA showed an inhibitory effect on the release of all pro-inflammatory cytokines, whereas the secretion of IL10 was rather potentiated (Fig. 2b). These observations resemble the results obtained at transcriptional 

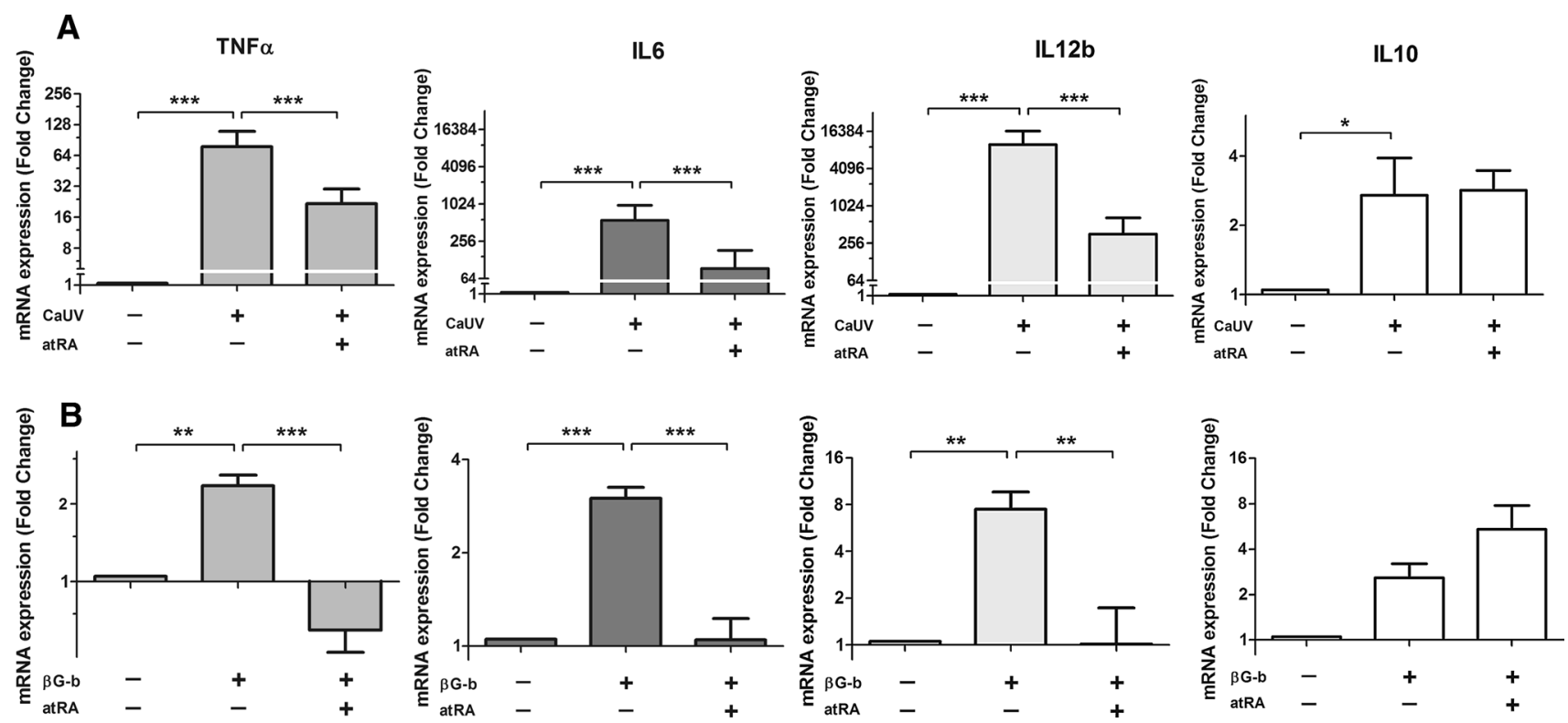

Fig. 1 Relative mRNA expression levels of TNF $\alpha$, IL6 and IL12b measured by qPCR. Monocytes were stimulated with either a UVtreated $C$. albicans yeasts (UV-Ca) or b $\beta$-1,3-glucan beads ( $\beta \mathrm{G}-\mathrm{b}$ ) for $5 \mathrm{~h}$ in the presence or absence of $1 \mu \mathrm{M}$ atRA. Both the $\beta-1,3-$ glucan-induced and the $C$. albicans-mediated up-regulation of pro-

inflammatory cytokine expression was significantly attenuated by atRA. Data were obtained from five independent experiments, each performed with cells from different donors. Results are presented as mean \pm SEM of the fold change relative to the control (unstimulated cells). $* * * p \leq 0.001$

level and suggest a strong anti-inflammatory role of vitamin A in fungal infections.

\section{Retinoic acid modulates the expression of Dectin-1}

Since we could observe an impact of vitamin A on the function of Dectin-1 in terms of the receptor-mediated cytokine production, we next wanted to investigate whether vitamin A had an effect on the expression of this PRR. Under the experimental settings of our stimulation assay, the expression of Dectin-1 was measured at transcriptional level after 5 and $16 \mathrm{~h}$ of incubation with $C$. albicans. At both time points, atRA supplementation led to a decreased expression of Dectin-1 mRNA. Moreover, the inhibitory effect of atRA seemed to increase over time, reaching a fivefold down-regulation of Dectin-1 mRNA after $16 \mathrm{~h}$ of incubation with $C$. albicans (Fig. 3a). Similar down-regulation was also observed when cells were stimulated with atRA alone, in the absence of fungal challenge (Suppl. Fig. 2). Interestingly, C. albicans itself was also able to slightly dampen the expression of Dectin-1 at transcriptional level (Fig. 3a).

Next, we explored the expression of Dectin- 1 on the cell surface of the monocytes. As shown in Fig. 3b, our findings at transcriptional level could be reproduced at protein level by flow cytometry. A shift in the MFI became apparent after C. albicans stimulation and reached its maximum drop in the presence of atRA (Fig. 3b). To further assess the impact of atRA on Dectin-1 over time, relative MFI values were

calculated for each time point. In agreement with the observations at transcriptional level, the atRA-mediated downregulation of Dectin-1 increased over time as assessed by a trend test (Page's $L$ test; $p<0.001$; Fig. 3c). These results suggest a sustained modulation of the anti-fungal response by vitamin A. Moreover, when we analyzed the influence of atRA on the expression pattern of two known co-receptors of Dectin-1, TLR2 and Galectin-3, a highly significant down-regulation could be observed in both cases (Fig. 3d). While $C$. albicans increased the mRNA expression of both TLR2 and Galectin-3, this up-regulation was almost completely abrogated in the presence of atRA.

RAR-dependent and RAR-independent mechanisms mediate the atRA-induced anti-inflammatory effect in monocytes

To determine the involvement of specific nuclear receptors in our findings, we first characterized the expression profiles of all possible RARs in our monocytes by RT-PCR. We could not detect any expression of RAR $\beta$, but obtained a clear signal for the expression of both RAR $\alpha$ and RAR $\gamma$ mRNA in all five monocyte samples used in this study (Fig. 4a). To define the relevance of these two receptors in the atRA-mediated anti-inflammatory effect, specific agonists and antagonists of each RAR were used in our experimental setting.

When the monocytes were pre-incubated with the specific RAR agonists, both RAR $\alpha$ agonist BMS753 and 


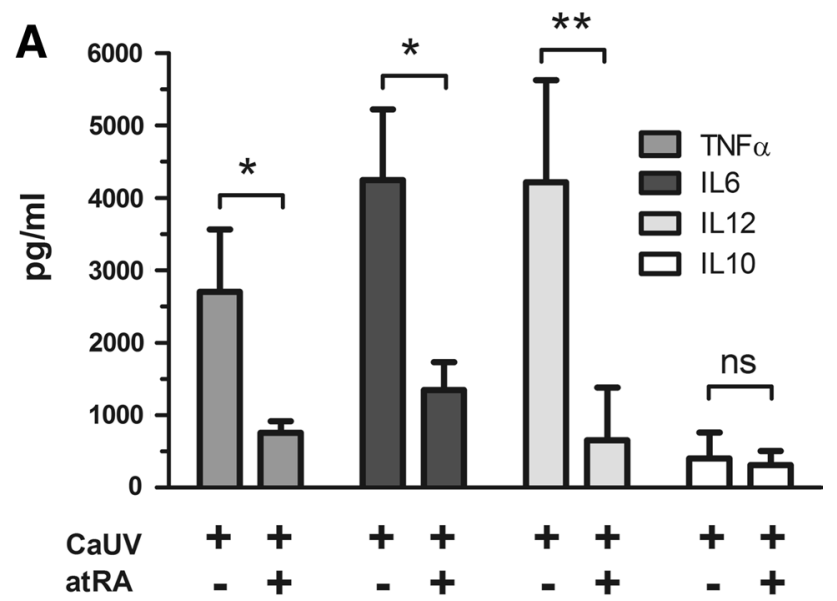

B

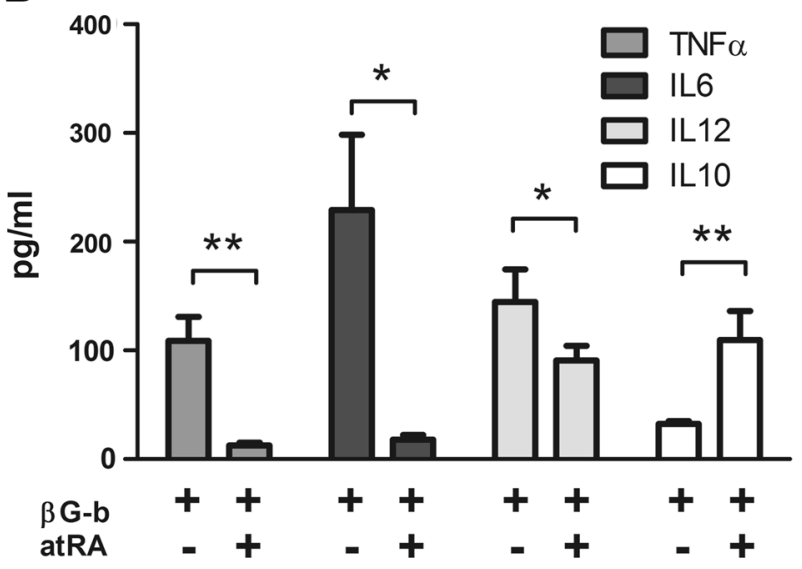

Fig. 2 Cytokine measurement in the culture supernatants. Monocytes were stimulated with either a UV-treated $C$. albicans yeasts (UV-Ca) or b $\beta$-1,3-glucan beads ( $\beta \mathrm{G}-\mathrm{b})$ in the presence or absence of $1 \mu \mathrm{M}$ atRA. After $16 \mathrm{~h}$ culture supernatants were analyzed using specific ELISAs for TNF $\alpha$, IL6, IL12 and IL10. In the presence of atRA, the secretion of all pro-inflammatory cytokines was significantly inhibited. Columns and error bars represent the mean \pm SEM of five independent experiments. $* p \leq 0.05 ; * * p \leq 0.01$

RAR $\gamma$ agonist BMS961 were able to mimic the repressive effect of atRA, although only partially in most cases (Fig. 4b). These results suggest that both RAR receptors are capable to mediate the observed down-regulation of the inflammatory response against $C$. albicans. To further prove their involvement in our particular experimental setting, specific antagonists of both RARs were used to test whether the expression levels of each gene could be restored in their presence. For both TNF $\alpha$ and IL6, the C. albicans-induced expression levels could be fully restored in the presence of the RAR antagonists in a significant manner (Fig. 4b). Also the expression of IL12b could be restored significantly, but only partially. In contrast, the atRA-mediated down-regulation of Dectin-1 mRNA was not affected by the addition of the RAR antagonists
(Fig. 4b). Taken together, these results suggest a RARdependent mechanism for the atRA-mediated modulation of the pro-inflammatory cytokines, whereas an additional RAR-independent mechanism seems to play a major role in the transcriptional regulation of Dectin-1.

\section{Discussion}

Despite the increasing interest in the immunomodulatory role of vitamin A, no evidence has been reported addressing the impact of vitamin A on the immune response to fungi. In the present study, we have characterized the effect of atRA on the $C$. albicans-induced immune response in human monocytes. Our results show a strong immunomodulatory role for atRA, leading to a highly significant suppression of the fungi-induced expression of TNF $\alpha$, IL6 and IL12. This down-regulation could be assessed at both transcriptional and post-translational level.

In fungal infections, the immune response is initiated after recognition of the pathogen by specific PRRs, such as Dectin-1 [26]. To address the impact of vitamin A on this important receptor in the fungal immune activation, we stimulated the monocytes using $\beta$-1,3-glucan beads as specific Dectin-1 ligand. Under these conditions, the modulatory effect of atRA resembled the one observed in experiments performed with $C$. albicans. Besides the impact of atRA on the functional output of Dectin-1 activation, we investigated the effect of atRA on the expression of the receptor upon $C$. albicans challenge. Interestingly, $C$. albicans alone was able to down-regulate the expression of Dectin-1, which has not been described previously in the literature. This observation might suggest a new mechanism to be included in the growing record of immuneescaping strategies described for this fungus [34, 35]. When atRA was added in this experimental setting, an even stronger inhibitory impact on the expression of Dectin-1 could be observed. The atRA-mediated down-regulation of the receptor could be assessed at transcriptional level and by flow cytometry. The inhibitory effect of atRA was not challenge-dependent, since a similar effect could be observed in the absence of $C$. albicans (Suppl. Fig. 2). This effect seems also not to be related to a process of atRAinduced differentiation, since a comparable down-regulation of Dectin-1 could be observed when terminally differentiated dendritic cells were stimulated with atRA (data not shown). Moreover, we could demonstrate that the suppressive effect of atRA increased over time, which suggests that the immunomodulatory impact of vitamin A might be sustained over a prolonged period. In addition, the known Dectin-1 co-receptors TLR2 [36] and Galectin-3 [37], which have been shown to enhance the Dectin-1-dependent immune response, were also down-regulated by atRA. 
Our results raised the question whether the observed drop in pro-inflammatory cytokine production could be explained by the atRA-mediated down-regulation of Dectin-1 and its co-receptors. The effect of atRA on the cytokine expression could be observed at transcriptional level after $5 \mathrm{~h}$ of incubation. Interestingly, at this stage, the atRA-mediated Dectin-1 down-regulation at the cell surface was already apparent, although only in a very incipient manner (Suppl. Fig. 3). Therefore, we cannot exclude that the down-regulation of the receptor might contribute, at least in part, to the observed drop in cytokine levels. Nevertheless, it is likely that other direct mechanisms play a prominent role in the early phase of the atRA-mediated immunomodulation. This is supported by our observation that short pre-incubation periods with atRA led to a stronger down-regulation of the fungi-induced cytokine expression than pre-incubation periods of $24 \mathrm{~h}$, when the Dectin-1-down-regulation on the cell surface reached a maximum (Suppl. Fig. 4). This is also in agreement with the observation that atRA has been described to modulate the immune response to LPS, a Dectin-1-independent immunological challenge, at least in other cell types such as dendritic cells and macrophages [16].

In monocytes, the immunomodulatory role of atRA on the immune response against LPS has been reported by Oeth et al. [38] and Wang et al. [39]. While Oeth et al. could not detect any atRA-mediated changes in the TNF $\alpha$ secretion after stimulation with LPS, Wang et al. observed only a very slight impact of atRA ( $<$ twofold change) on the LPS-induced TNF $\alpha$ and IL12 mRNA expression levels. In our study, the addition of atRA led to an almost $100 \%$ abrogation of the Dectin-1-mediated expression and secretion of TNF $\alpha$, IL6 and IL12 in human monocytes. It would be interesting to investigate whether the higher impact of atRA observed in our study could be related to the nature of the immunological challenge. The signal transduction of the LPS/TLR4 axis differs consistently from that of the Dectin-1-activation [26]. It is tempting to speculate that different PRR-signaling pathways might display different sensitivities to atRA-modulation. Further studies are required to comprehensively elucidate the mechanisms of atRAmediated modulation of the immune response.
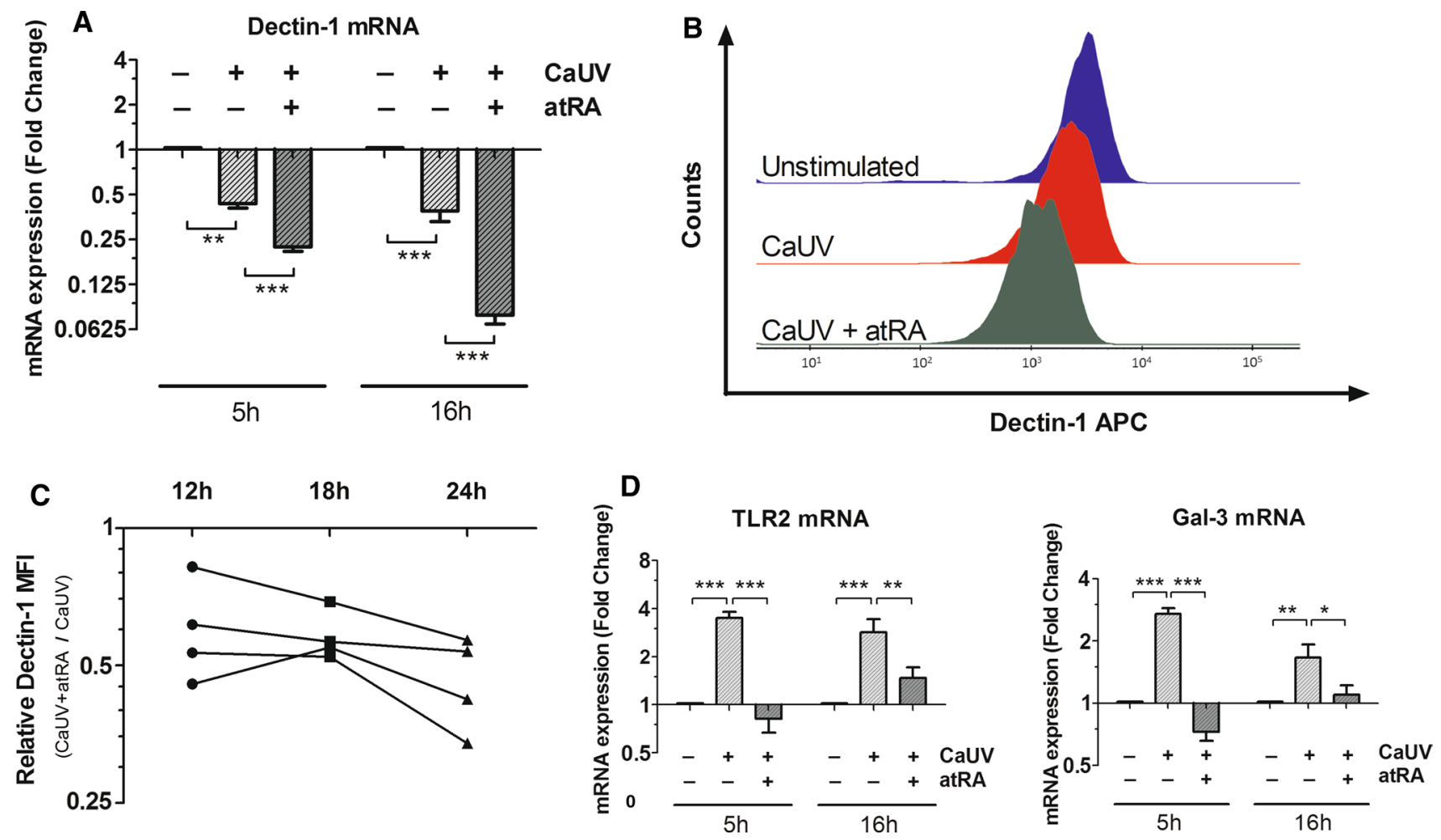

Fig. 3 Modulation of the Dectin-1-expression by retinoic acid upon C. albicans infection. Monocytes were stimulated with UV-treated $C$. albicans (CaUV) yeasts in the presence or absence of $1 \mu \mathrm{M}$ atRA. a Dectin-1-mRNA expression was measured after 5 and $16 \mathrm{~h}$. b Representative flow cytometry plot of Dectin-1-APC after $24 \mathrm{~h}$ of stimulation. c Relative Dectin-1 MFI over time. The relative MFI is defined as the MFI of the cells challenged with $C$. albicans in the presence of atRA divided by the MFI of the monocytes challenged with the fungi in the absence of atRA. d mRNA expression of Dectin-1 coreceptors TLR2 and Galectin-3 after 5 and $16 \mathrm{~h}$ of stimulation. Data for the transcriptional analysis were obtained from five independent experiments, each performed with cells from different donors. Results are presented as mean \pm SEM of the fold change relative to the control (unstimulated cells). For flow cytometry analysis, data from four independent experiments were collected. * $p \leq 0.05$; ** $p \leq 0.01$; $* * * p \leq 0.001$ 
A

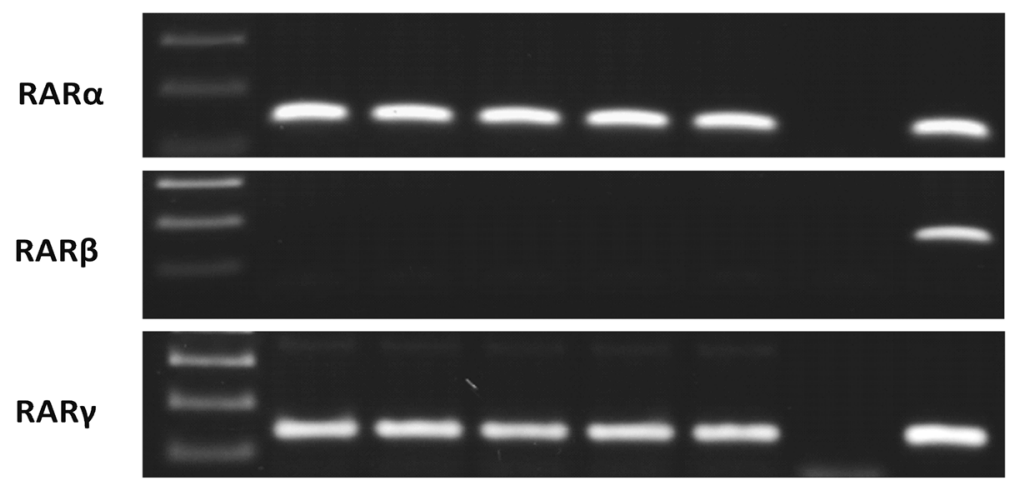

$145 \mathrm{bp}$

$158 \mathrm{bp}$

$137 \mathrm{bp}$
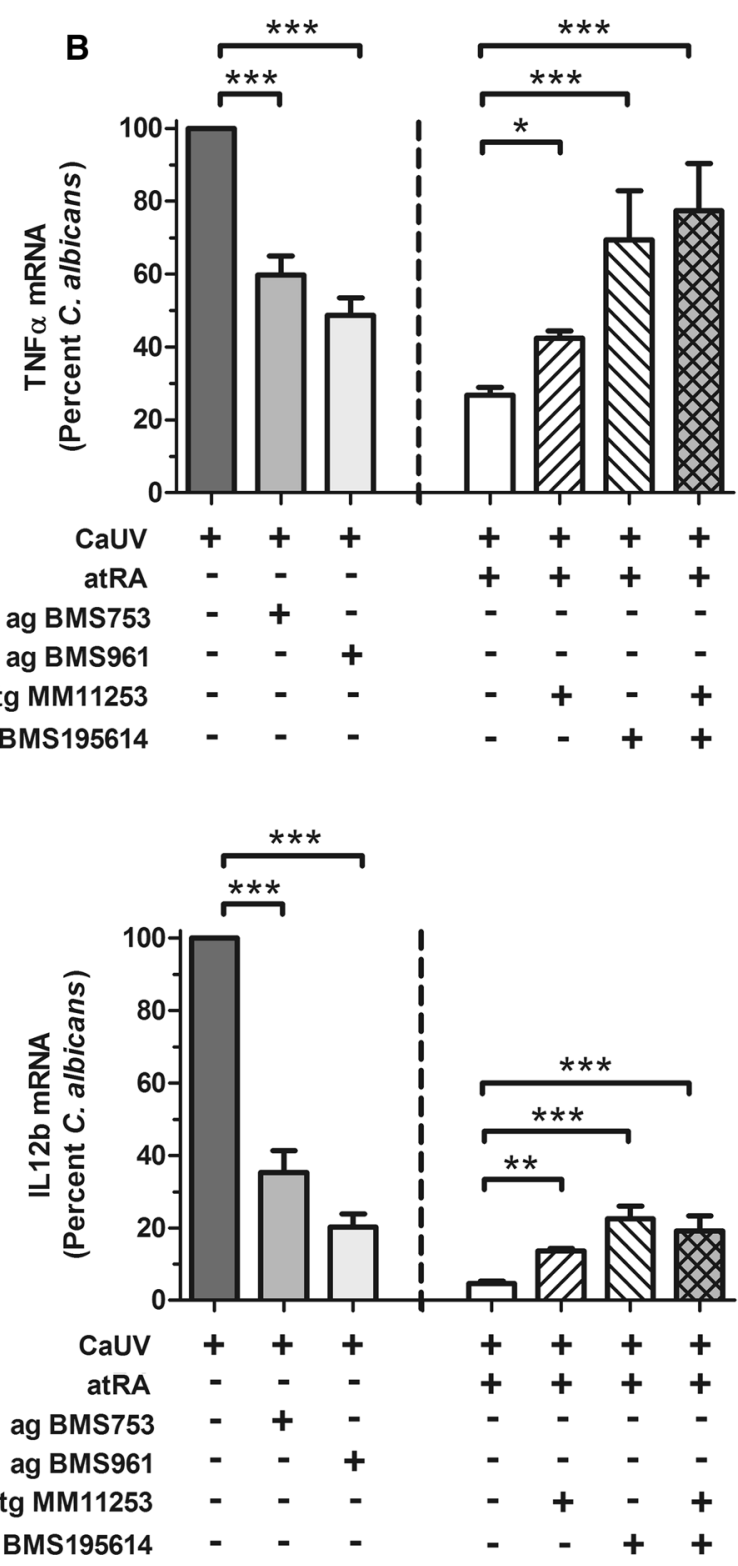

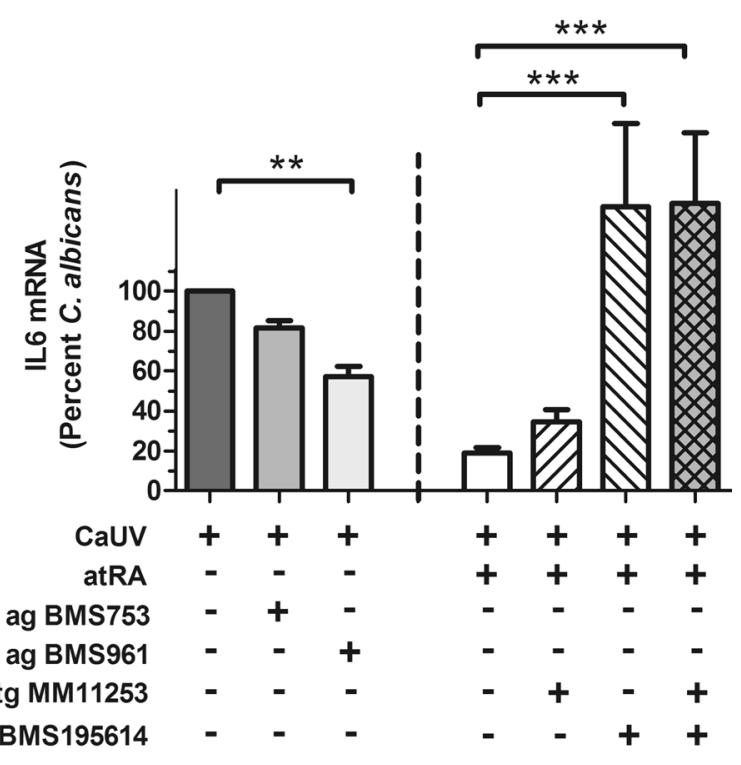

antg BMS195614 
4Fig. 4 Expression profile and immunomodulatory activity of retinoic acid receptors in human monocytes. a Transcriptional expression of RAR $\alpha$, RAR $\beta$ and RAR $\gamma$ in monocytes (samples M1-M5) as discriminated by agarose gel electrophoresis of PCR products. HEK293 cells $(\mathrm{RAR} \alpha), \mathrm{A} 549$ cells $(\mathrm{RAR} \beta)$ or NHBE cells $(\mathrm{RAR} \gamma)$ served as positive controls in each case. (RT- $=$ reverse transcription negative control) b Immunomodulatory effect of specific RAR-agonists BMS753 and BMS961 on the expression of TNF $\alpha$, IL6, IL12b and Dectin-1 upon $C$. albicans infection. Also shown is the inhibitory power of the specific RAR antagonists MM11253 and BMS195614 on the atRAmediated modulation of these genes. Monocytes were pre-incubated for $30 \mathrm{~min}$ with either RAR-specific agonists or atRA (in the presence or absence of RAR antagonists) and then challenged with UV-treated $C$. albicans yeasts (CaUV). Columns represent the percentage of the $C$. albicans-induced mRNA expression in each case. Data were collected from five independent experiments. ${ }^{*} \mathrm{p} \leq 0.05$; ** $p \leq 0.01 ; * * * p \leq 0.001$

Most of the biological actions of atRA are exerted through binding its specific nuclear receptors: RAR $\alpha$, $\operatorname{RAR} \beta$ and/or RAR $\gamma$ [40]. We investigated the expression of all three RARs in monocytes and could detect the expression of both RAR $\alpha$ and $\operatorname{RAR} \gamma$ mRNA, which is in agreement with previous studies [41]. The use of specific agonists and antagonists for each RAR allowed us to verify that both receptors are involved in the suppressive effects observed on TNF $\alpha$, IL6 and IL12. On the other hand, the vitamin A modulation of the Dectin-1 expression seemed to occur mainly through a RAR-independent mechanism.

The impact of vitamins on immunity and inflammation has been widely investigated in allergy and bacterial infections [42], but to a much lesser extent in fungal infections. Nevertheless, vitamin D has already been described to possess significant anti-inflammatory properties in Candida infections [43]. Meanwhile, the role of vitamin A in invasive candidiasis has remained unknown. Moreover, only very few studies have been conducted to investigate the vitamin A-status in septic patients, were $C$. albicans is a common nosocomial pathogen [44, 45]. Indeed, 33-55\% of all episodes of candidemia have been shown to occur in intensive care units (ICU) [46]. Such ICU-acquired candidemia in critically ill patients is associated with a particular high mortality of $>50 \%$ [47]. The search for anti-inflammatory immunomodulators is of particular importance in invasive candidiasis, where the dysregulation of the immune response rather than the pathogen has been shown to be responsible for the fatal host damage [22, 43]. Interestingly, a recent study reported an important inadequacy of retinol and $\beta$-carotene in a cohort of sepsis patients [45]. Thus, monitoring the serum levels of vitamin A and its adequate supplementation in individuals admitted in ICUs might have far-reaching prophylactic implications.

In conclusion, in this study, we have demonstrated a strong immunomodulatory role of vitamin $\mathrm{A}$ on the innate host response to $C$. albicans in human monocytes. In addition, we could show that atRA modulates both the function and the expression of Dectin-1. Moreover, the modulation of this PRR seems to increase over time, leading to a sustained regulation of the immune response. The observed effect of atRA on the cytokine production is likely to occur via activation of either or both $\operatorname{RAR} \alpha$ and $\operatorname{RAR} \gamma$. This study opens a new avenue to explore the role of vitamin $\mathrm{A}$ in fungal infections and to elucidate further molecular mechanisms of its immunomodulatory function.

Acknowledgments We want to thank Simone Tänzer for excellent technical assistance. This work was funded by the German Research Foundation (Collaborative Research Center/Transregio 124-Pathogenic fungi and their human host: Networks of Interaction, Project A5), by the German Federal Ministry of Education and Research (BMBF 03Z2JN22 to H.S. and 01EO1002 to A.H. and E.K./Center for Sepsis Control and Care), and the grants NIH/NIAID 1K08AI110655 to M.K.M., 1R01AI092084 to J.M.T. and 1R01AI097519 to J.M.V.

Conflict of interest The authors declare that they have no conflict of interest.

Ethical standards This work complies with the current laws of Germany.

Open Access This article is distributed under the terms of the Creative Commons Attribution License which permits any use, distribution, and reproduction in any medium, provided the original author(s) and the source are credited.

\section{References}

1. D'Ambrosio DN, Clugston RD, Blaner WS (2011) Vitamin A metabolism: an update. Nutrients 3(1):63-103. doi:10.3390/ nu3010063

2. Wolf G (1996) A history of vitamin A and retinoids. FASEB J 10(9):1102-1107

3. Wolf G (2007) Identification of a membrane receptor for retinolbinding protein functioning in the cellular uptake of retinol. Nutr Rev 65(8 Pt 1):385-388

4. Hall JA, Grainger JR, Spencer SP, Belkaid Y (2011) The role of retinoic acid in tolerance and immunity. Immunity 35(1):13-22. doi:10.1016/j.immuni.2011.07.002

5. Theodosiou M, Laudet V, Schubert M (2010) From carrot to clinic: an overview of the retinoic acid signaling pathway. Cell Mol Life Sci 67(9):1423-1445. doi:10.1007/s00018-010-0268-z

6. Clagett-Dame M, Knutson D (2011) Vitamin A in reproduction and development. Nutrients 3(4):385-428. doi:10.3390/nu3040385

7. Mark M, Ghyselinck NB, Chambon P (2009) Function of retinoic acid receptors during embryonic development. Nucl Recept Signal 7:e002. doi:10.1621/nrs.07002

8. Sommer A, Vyas KS (2012) A global clinical view on vitamin A and carotenoids. Am J Clin Nutr 96(5):1204s-1206s. doi:10.3945 /ajcn.112.034868

9. Glasziou PP, DE Mackerras (1993) Vitamin A supplementation in infectious diseases: a meta-analysis. BMJ 306(6874):366-370 (Clinical research ed)

10. Pino-Lagos K, Guo Y, Noelle RJ (2010) Retinoic acid: a key player in immunity. BioFactors 36(6):430-436. doi:10.1002/biof.117 (Oxford, England)

11. Israel H, Odziemiec C, Ballow M (1991) The effects of retinoic acid on immunoglobulin synthesis by human cord blood mononuclear cells. Clin Immunol Immunopathol 59(3):417-425 
12. Ross AC, Chen Q, MaY (2011) Vitamin A and retinoic acid in the regulation of B-cell development and antibody production. Vitam Horm 86:103-126. doi:10.1016/b978-0-12-386960-9.00005-8

13. Worm M, Krah JM, Manz RA, Henz BM (1998) Retinoic acid inhibits CD40 + interleukin-4-mediated IgE production in vitro. Blood 92(5): 1713-1720

14. Dawson HD, Collins G, Pyle R, Key M, Weeraratna A, Deep-Dixit V, Nadal CN, Taub DD (2006) Direct and indirect effects of retinoic acid on human Th2 cytokine and chemokine expression by human T lymphocytes. BMC Immunol 7:27. doi:10.1186/1471-2172-7-27

15. Tsai YC, Chang HW, Chang TT, Lee MS, Chu YT, Hung CH (2008) Effects of all-trans retinoic acid on Th1- and Th2-related chemokines production in monocytes. Inflammation 31(6):428433. doi:10.1007/s10753-008-9095-x

16. Wojtal KA, Wolfram L, Frey-Wagner I, Lang S, Scharl M, Vavricka SR, Rogler G (2013) The effects of vitamin A on cells of innate immunity in vitro. Toxicol In Vitro 27(5):1525-1532. doi:10.1016/j.tiv.2013.03.013

17. Villamor E, Fawzi WW (2000) Vitamin A supplementation: implications for morbidity and mortality in children. J Infect Dis 182(Suppl 1):S122-S133. doi:10.1086/315921

18. Semba RD (1999) Vitamin A and immunity to viral, bacterial and protozoan infections. Proc Nutr Soc 58(3):719-727

19. Lamagni TL, Evans BG, Shigematsu M, Johnson EM (2001) Emerging trends in the epidemiology of invasive mycoses in England and Wales (1990-9). Epidemiol Infect 126(3):397-414

20. Lewis RE (2009) Overview of the changing epidemiology of candidemia. Curr Med Res Opin 25(7):1732-1740. doi:10.1185/03007990902990817

21. Pfaller MA, Diekema DJ (2007) Epidemiology of invasive candidiasis: a persistent public health problem. Clin Microbiol Rev 20(1):133-163. doi:10.1128/cmr.00029-06

22. Majer O, Bourgeois C, Zwolanek F, Lassnig C, Kerjaschki D, Mack M, Muller M, Kuchler K (2012) Type I interferons promote fatal immunopathology by regulating inflammatory monocytes and neutrophils during Candida infections. PLoS Pathog 8(7):e1002811. doi:10.1371/journal.ppat.1002811

23. Netea MG, Brown GD, Kullberg BJ, Gow NA (2008) An integrated model of the recognition of Candida albicans by the innate immune system. Nat Rev Microbiol 6(1):67-78. doi:10.10 38/nrmicro 1815

24. Ngo LY, Kasahara S, Kumasaka DK, Knoblaugh SE, Jhingran A, Hohl TM (2014) Inflammatory monocytes mediate early and organ-specific innate defense during systemic candidiasis. J Infect Dis 209(1):109-119. doi:10.1093/infdis/jit413

25. Netea MG, Gijzen K, Coolen N, Verschueren I, Figdor C, Van der Meer JW, Torensma R, Kullberg BJ (2004) Human dendritic cells are less potent at killing Candida albicans than both monocytes and macrophages. Microbes Infect 6(11):985-989. doi:10.1016/j.micinf.2004.05.013

26. Drummond RA, Brown GD (2011) The role of Dectin-1 in the host defence against fungal infections. Curr Opin Microbiol 14(4):392-399. doi:10.1016/j.mib.2011.07.001

27. Tam JM, Mansour MK, Khan NS, Yoder NC, Vyas JM (2012) Use of fungal derived polysaccharide-conjugated particles to probe Dectin-1 responses in innate immunity. Integr Biol 4(2):220-227. doi:10.1039/c2ib00089j

28. Mansour MK, Tam JM, Khan NS, Seward M, Davids PJ, Puranam S, Sokolovska A, Sykes DB, Dagher Z, Becker C, Tanne A, Reedy JL, Stuart LM, Vyas JM (2013) Dectin-1 activation controls maturation of beta-1,3-glucan-containing phagosomes. J Biol Chem 288(22):16043-16054. doi:10.1074/jbc.M113.473223

29. Gillum AM, Tsay EY, Kirsch DR (1984) Isolation of the Candida albicans gene for orotidine- $5^{\prime}$-phosphate decarboxylase by complementation of $S$. cerevisiae ura3 and E. coli pyrF mutations. Mol Gen Genet 198(1):179-182
30. Zuker M (2003) Mfold web server for nucleic acid folding and hybridization prediction. Nucleic Acids Res 31(13):3406-3415

31. Pfaffl MW (2001) A new mathematical model for relative quantification in real-time RT-PCR. Nucleic Acids Res 29(9):e45

32. Rieu I, Powers SJ (2009) Real-time quantitative RT-PCR: design, calculations, and statistics. Plant Cell 21(4):1031-1033. doi:10.1 105/tpc.109.066001

33. Pfaffl MW, Tichopad A, Prgomet C, Neuvians TP (2004) Determination of stable housekeeping genes, differentially regulated target genes and sample integrity: BestKeeper-Excel-based tool using pair-wise correlations. Biotechnol Lett 26(6):509-515

34. Cheng SC, Joosten LA, Kullberg BJ, Netea MG (2012) Interplay between Candida albicans and the mammalian innate host defense. Infect Immun 80(4):1304-1313. doi:10.1128/iai.06146-11

35. Zipfel PF, Skerka C, Kupka D, Luo S (2011) Immune escape of the human facultative pathogenic yeast Candida albicans: the many faces of the Candida Pra1 protein. Int $\mathrm{J}$ Med Microbiol 301(5):423-430. doi:10.1016/j.ijmm.2011.04.010

36. Ferwerda G, Meyer-Wentrup F, Kullberg BJ, Netea MG, Adema GJ (2008) Dectin-1 synergizes with TLR2 and TLR4 for cytokine production in human primary monocytes and macrophages. Cell Microbiol 10(10):2058-2066. doi:10.1111/j.1462-5822.2008.01188.x

37. Esteban A, Popp MW, Vyas VK, Strijbis K, Ploegh HL, Fink GR (2011) Fungal recognition is mediated by the association of dectin-1 and galectin-3 in macrophages. Proc Natl Acad Sci USA 108(34):14270-14275. doi:10.1073/pnas.1111415108

38. Oeth P, Yao J, Fan ST, Mackman N (1998) Retinoic acid selectively inhibits lipopolysaccharide induction of tissue factor gene expression in human monocytes. Blood 91(8):2857-2865

39. Wang X, Allen C, Ballow M (2007) Retinoic acid enhances the production of IL-10 while reducing the synthesis of IL-12 and TNF-alpha from LPS-stimulated monocytes/macrophages. J Clin Immunol 27(2):193-200. doi:10.1007/s10875-006-9068-5

40. Laudet V, Gronemeyer H (2002) The nuclear receptor facts book. Academic, San Diego

41. Fritsche J, Stonehouse TJ, Katz DR, Andreesen R, Kreutz M (2000) Expression of retinoid receptors during human monocyte differentiation in vitro. Biochem Biophys Res Commun 270(1):17-22. doi:10.1006/bbrc.2000.2371

42. Shaik-Dasthagirisaheb YB, Varvara G, Murmura G, Saggini A, Caraffa A, Antinolfi P, Tete S, Tripodi D, Conti F, Cianchetti E, Toniato E, Rosati M, Speranza L, Pantalone A, Saggini R, Tei M, Speziali A, Conti P, Theoharides TC, Pandolfi F (2013) Role of vitamins $\mathrm{D}, \mathrm{E}$ and $\mathrm{C}$ in immunity and inflammation. J Biol Regul Homeost Agents 27(2):291-295

43. Khoo AL, Chai LY, Koenen HJ, Kullberg BJ, Joosten I, van der Ven AJ, Netea MG (2011) 1,25-dihydroxyvitamin D3 modulates cytokine production induced by Candida albicans: impact of seasonal variation of immune responses. J Infect Dis 203(1):122130. doi:10.1093/infdis/jiq008

44. Otto GP, Sossdorf M, Claus RA, Rodel J, Menge K, Reinhart K, Bauer M, Riedemann NC (2011) The late phase of sepsis is characterized by an increased microbiological burden and death rate. Crit Care 15(4):R183. doi:10.1186/cc10332 (London, England)

45. Ribeiro Nogueira C, Ramalho A, Lameu E, Da Silva Franca CA, David C, Accioly E (2009) Serum concentrations of vitamin A and oxidative stress in critically ill patients with sepsis. Nutr Hosp 24(3):312-317

46. Bouza E, Munoz P (2008) Epidemiology of candidemia in intensive care units. Int J Antimicrob Agents 32(Suppl 2):S87-S91. doi:10.1016/s0924-8579(08)70006-2

47. de Gonzalez Molina FJ, Leon C, Ruiz-Santana S, Saavedra P (2012) Assessment of candidemia-attributable mortality in critically ill patients using propensity score matching analysis. Crit Care 16(3):R105. doi:10.1186/cc11388 (London, England) 